\title{
TURÁN FUNCTION AND $H$-DECOMPOSITION PROBLEM FOR GEM GRAPHS
}

\author{
HENRY LIU \\ School of Mathematics and Statistics \\ Central South University \\ Changsha 410083, China \\ e-mail: henry-liu@csu.edu.cn \\ AND \\ Teresa Sousa \\ Escola Naval and Centro de Investigação Naval \\ Escola Naval - Alfeite \\ 2810-001 Almada, Portugal \\ and \\ Centro de Matemática e Aplicações \\ Faculdade de Ciências e Tecnologia \\ Universidade Nova de Lisboa \\ Campus de Caparica \\ 2829-516 Caparica, Portugal \\ e-mail: teresa.maria.sousa@marinha.pt
}

\begin{abstract}
Given a graph $H$, the Turán function $\operatorname{ex}(n, H)$ is the maximum number of edges in a graph on $n$ vertices not containing $H$ as a subgraph. For two graphs $G$ and $H$, an $H$-decomposition of $G$ is a partition of the edge set of $G$ such that each part is either a single edge or forms a graph isomorphic to $H$. Let $\phi(n, H)$ be the smallest number $\phi$ such that any graph $G$ of order $n$ admits an $H$-decomposition with at most $\phi$ parts. Pikhurko and Sousa conjectured that $\phi(n, H)=\operatorname{ex}(n, H)$ for $\chi(H) \geq 3$ and all sufficiently large $n$. Their conjecture has been verified by Özkahya and Person for all edge-critical graphs $H$. In this article, we consider the gem graphs gem $_{4}$ and gem $_{5}$. The graph gem 4 consists of the path $P_{4}$ with four vertices $a, b, c, d$ and edges $a b, b c, c d$ plus a universal vertex $u$ adjacent to $a, b, c, d$, and the graph gem $_{5}$ is similarly defined with the path $P_{5}$ on five vertices. We determine
\end{abstract}


the Turán functions ex $\left(n, \operatorname{gem}_{4}\right)$ and ex $\left(n, \operatorname{gem}_{5}\right)$, and verify the conjecture of Pikhurko and Sousa when $H$ is the graph gem ${ }_{4}$ and gem ${ }_{5}$.

Keywords: gem graph, Turán function, extremal graph, graph decomposition.

2010 Mathematics Subject Classification: 05C35, $05 \mathrm{C} 70$.

\section{REFERENCES}

[1] P. Allen, J. Böttcher and Y. Person, An improved error term for minimum $\mathrm{H}$ decompositions of graphs, J. Combin. Theory Ser. B 108 (2014) 92-101. doi:10.1016/j.jctb.2014.03.001

[2] B. Bollobás, On complete subgraphs of different orders, Math. Proc. Cambridge Philos. Soc. 79 (1976) 19-24. doi:10.1017/S0305004100052063

[3] D. Dor and M. Tarsi, Graph decomposition is NP-complete: a complete proof of Holyer's conjecture, SIAM J. Comput. 26 (1997) 1166-1187. doi:10.1137/S0097539792229507

[4] P. Erdős, A.W. Goodman and L. Pósa, The representation of a graph by set intersections, Canad. J. Math. 18 (1966) 106-112. doi:10.4153/CJM-1966-014-3

[5] R. Faudree and R. Schelp, Path Ramsey numbers in multicolorings, J. Combin. Theory Ser. B 19 (1975) 150-160. doi:10.1016/0095-8956(75)90080-5

[6] H. Liu and T. Sousa, Decompositions of graphs into fans and single edges, J. Graph Theory 85 (2017) 400-411.

doi:/10.1002/jgt.22069

[7] L. Özkahya and Y. Person, Minimum H-decompositions of graphs: edge-critical case, J. Combin. Theory Ser. B 102 (2012) 715-725. doi:10.1016/j.jctb.2011.10.004

[8] O. Pikhurko, A note on the Turán function of even cycles, Proc. Amer. Math. Soc. 140 (2012) 3687-3692.

doi:10.1090/S0002-9939-2012-11274-2

[9] O. Pikhurko and T. Sousa, Minimum H-decompositions of graphs, J. Combin. Theory Ser. B 97 (2007) 1041-1055. doi:10.1016/j.jctb.2007.03.002

[10] T. Sousa, Decompositions of graphs into 5-cycles and other small graphs, Electron. J. Combin. 12 (2005) \#R49.

[11] T. Sousa, Decompositions of graphs into a given clique-extension, Ars Combin. 100 (2011) 465-472. 
[12] T. Sousa, Decompositions of graphs into cycles of length seven and single edges, Ars Combin. 119 (2015) 321-329.

[13] P. Turán, On an extremal problem in graph theory, Mat. Fiz. Lapok 48 (1941) 436-452.

Received 10 October 2016

Revised 26 January 2017

Accepted 2 February 2017 